\title{
Local activation of the pulmonary extravascular angiotensin system induces epithelial apoptosis and lung fibrosis
}

\begin{abstract}
Previous work suggests that a local extravascular angiotensin system plays an important role in the development of pulmonary fibrosis through stimulation of alveolar epithelial cell (AEC) apoptosis and collagen deposition. To demonstrate a causative role for the local tissue angiotensin (ANG) system in lung fibrosis, we hypothesize that overexpression of the angiotensinogen (AGT) gene or pharmacologic elevation of lung tissue ANG II levels might cause apoptosis of AECs and lung fibrosis. ANGII levels were elevated in rat or mouse lung tissue by intratracheal instillation of either purified ANGII or an adenovirus expressing AGT, or by ubiquitous overexpression of AGT in transgenic mice. Intratracheal instillation of purified ANGII caused significant collagen accumulation in lung tissue, both ex vivo and in vivo. Ubiquitous overexpression of AGT enhanced the profibrotic effect of bleomycin given at suboptimal doses. Intratracheal delivery of an adenoviral vector expressing mouse AGT (Ad-AGT) overexpressed AGT primarily in AECs and caused both apoptosis of AECs and pulmonary fibrosis. The lung collagen accumulation and AEC apoptosis caused by Ad-AGT was blocked by the caspase inhibitor ZVAD-fmk, by the ANG receptor AT1 antagonist Losartan or by the non-selective ANGII receptor antagonist Saralasin. Together, these data support the hypothesis that elevated pulmonary expression of AGT and its conversion to angiotensin II plays a causative role in the development of lung fibrosis through its induction of AEC apoptosis.
\end{abstract}

Keywords: angiotensinogen, alveolar epithelium, lung fibrogenesis
Volume 5 Issue 6 - 2018

\author{
Xiaopeng Li,' Jiaju Zhuang,' Bruce D Uhal' \\ 'Department of Pediatrics, Michigan State University, USA \\ ${ }^{2}$ Department of Physiology, Bethune Military Medical College, \\ China
}

Correspondence: Bruce D Uhal, Department of Physiology, Michigan State University, 3197 Biomedical and Physical Sciences Building, East Lansing, MI 48824, USA, Tel (5I7) 884-5I 3 I, Fax (5 I7) 355-5I25, Email uhal@msu.edu

Received: December 05, 2018 | Published: December 18, 2018

\section{Introduction}

Idiopathic Pulmonary fibrosis (IPF) is a pathological condition resulting from injury to the lung and an ensuing fibrotic response leading to thickening of the alveolar walls and the obliteration of the alveolar space without known etiology. ${ }^{1}$ Unfortunately, the treatment of IPF has not been successful since the pathogenesis of IPF is not clear. $^{2,3}$

Studies in our lab and others using BLEO-induced rat and mouse PF models strongly suggested a role of epithelial apoptosis as the profibrotic event in fibrogenesis. The evidences include: First, apoptosis of was found in both patients with $\mathrm{IPF}^{4}$ and animal IPF models. ${ }^{5}$ Second, induction of apoptosis in the epithelium is sufficient to initiate a fibrotic response. ${ }^{6}$ Third, blockade of the apoptosis of AECs could prevent fibrotic response. ${ }^{7,8}$ These studies support the hypothesis that IPF results from the epithelial injury and failure to reepithelization. ${ }^{9}$ Results obtained from studies on human lungs are consistent with those on animal models. ${ }^{4,10,11}$

Previous work suggests that pulmonary angiotensin system plays an important role in the development of pulmonary fibrosis. Marshall and our data showed that pulmonary ANG II is elevated in bleomycin-induced pulmonary fibrosis model. ${ }^{12,13}$ Our recently results demonstrated that AGT, the only precursor of ANG II, is increased in the type II AECs (AECs) and myofibroblasts at both mRNA level and protein level, and its expression is related with apoptosis of AECs and required in the development of bleomycin-induced pulmonary fibrosis, ${ }^{12}$ and similar finding was observed in IPF patient. ${ }^{14}$ In addition, the converting enzymes for synthesis of angiotensin peptides like Cathepsin $\mathrm{D},{ }^{15,16}$ angiotensin converting enzyme (ACE) ${ }^{17,18}$ and $\mathrm{AT} 1^{19,20}$ receptors were upregulated in both animal and human fibrotic lung. Furthermore, Angiotensin system antagonists like ACE inhibitors and AT1 selective antagonists blocked experimental lung fibrosis. ${ }^{8,21-25}$ However, the causative effect of pulmonary angitensin system on pulmonary fibrosis is not clear. Therefore, we hypothesize that overexpression of AGT or elevation of pulmonary ANG II level in the lung will cause apoptosis of AECs and pulmonary fibrosis.

In the present study, we demonstrated that purified ANG II was administrated to elevate the local ANG II level in the lung, and caused significant collagen accumulation in the lungs both ex vivo and in vivo. IT delivery of Ad-AGT overexpressed AGT primarily in the lung and caused apoptosis of AECs and pulmonary fibrosis which can be blocked by Caspases inhibitor ZVAD, AT1 antagonist Losartan, and non-selective angiotensin II receptor antagonist Saralasin

\section{Materials and methods}

\section{Reagents and materials}

Purified adenoviruses (Vectors with or without AGT) were obtained from Vector Biolabs (Philadelphia, PA). Alkaline phosphatase conjugated streptavidin, were obtained from Boehringer Mannheim, Indianapolis, IN. BLEO was obtained from Sigma Chemical Co., Saint Louis, MO. Reagents for detection of alkaline phosphatase and other secondary reagents for ISEL of DNA or Western blotting were from sources described earlier. ${ }^{8}$ All other materials were of reagent grade and were obtained from Sigma Chemical Co. 


\section{Animals, induction of pulmonary fibrosis ex vivo and in vivo, and surgical procedures}

\section{Lung explant culture}

All explants were obtained from normal adult male Wistar rat lung that was PBS-perfused in situ before excision of the lungs. Explants of $1 \mathrm{~mm}^{2}$ were prepared by mincing of blood-depleted rat lung, and were cultured in Transwell polycarbonate inserts $(3.0-\mu \mathrm{m}$ pore; Costar, Corning, NY) under a thin layer $(1 \mathrm{~mm})$ of Dulbecco's modified Eagle's medium cell culture medium to facilitate gas exchange. ${ }^{26} \mathrm{After}$ excision of the lungs, treatment with purified ANG II or Saralasin was initiated ex vivo by intratracheal instillation of ANG II at $10^{-7}$ $\mathrm{M}$ in $2 \mathrm{ml}$ of sterile Dulbecco's modified Eagle's medium (+/- nonselective angiotensin receptor antagonist saralasin $\mathrm{SAR}=$ at $50 \mathrm{ug} / \mathrm{mL}$ ). The culture medium for explants also contained ANG II at $10^{-7} \mathrm{M},+/-$ $\mathrm{SAR}=$ at $50 \mathrm{ug} / \mathrm{mL}$ and Vitamin $\mathrm{C}(0.1 \mathrm{mM})$. The minced lung chunks were cultured in serum free medium with 1\% ITS (Elimination of the AGT from serum). 14 days after culture, explants were harvested by transfer into liquid $\mathrm{N} 2$ and storage at $-80^{\circ} \mathrm{C}$ until assay. Lung explants was processed for measurements of lung collagen by using total hydroxyproline (HP) assay.

\section{Induction of lung injury and fibrosis by purified ANG II administrated by I.T. or aerosol}

Adult male Wistar rats, $150-200 \mathrm{~g}$, were housed in a satellite facility of University Laboratory Animal Resources, Michigan State University. To test if purified ANG II can induce apoptosis of AECs in vivo which is hypothesized as the initial step of fibrogensis, as it did in vitro, male Wistar rats were instilled I.T. with either ANG II (10uM), ANG II plus the caspases inhibitor ZVADfmk (60uM), ANG II plus the AT1 receptor antagonist, losartan (LOS, 100uM) or PBS vehicle alone. 6 or 20 hours later, the lungs were lavaged and processed for paraffin-embedded sections on which TUNEL staining and anti-active form caspase 3 staining were conducted.

To test if purified ANG II can induce pulmonary fibrosis in vivo, repeated administration of purified ANG II was given by aerosol. 5 rats per group were exposed to aerosolized ANG II (10uM) or saline administrated by a nebulizer for 30 minutes, twice a day for 14 days. Whole left lungs were harvested for hydroxyproline (HP) assay to measure collagen amount.

\section{Induction of Lung Injury and Fibrosis by I.T. bleomycin in mice}

All mice were obtained from The Jackson Laboratories, Bar Harbor, ME, and were housed in a satellite facility of University Laboratory Animal Resources, Michigan State University. Control animals were wild-type C57BL/6J mice used at 7 to 8 weeks of age. Some experiments also used mice of the same genetic background but with systemic overexpression of AGT as those mice carry 4 copies of AGT gene and have a $25 \%$ increase of plasma AGT level compared to wild type (WT) mice. ${ }^{27}$ Animals under pentobarbital anesthesia received a single intratracheal instillation of bleomycin sulfate (BLEO) at of $0.2,0.6$, and $1 \mathrm{U} / \mathrm{kg}$ body weight, in $50 \mu \mathrm{l}$ of sterile saline. The degree of fibrosis was evaluated on days 14. Doses of 0.2 , $0.6 \mathrm{U} / \mathrm{kg}$ are suboptimal, but are important for this study, because the optimal dose of $1 \mathrm{U} / \mathrm{kg}$ may cause a saturating effect and thus mask the contribution of AGT to lung fibrosis.

The $50 \mu 1$ dose was instilled at end-expiration, and the liquid was followed immediately by $300 \mu$ of air to ensure delivery to the distal airways. Control animals were instilled with an equal volume of sterile saline by using previously published protocols. ${ }^{8} 14$ days after instillation of BLEO, at which point all animals were sacrificed for histology, detection of collagen.

\section{Induction of lung injury and fibrosis by I.T.Ad-AGT in mice}

Purified adenoviruses (Vectors with or without AGT) were obtained from Vector Biolabs (Philadelphia, PA). Intratracheal instillations of adenoviruses were performed on 6 to 8 weeks old C57BL/6 mice. The purified adenoviruses (Vectors with or without AGT) were instilled in doses of $10^{7}, 10^{8}$, and $10^{9}$ pfu per mouse. ${ }^{28}$ Animals were sacrificed at days $1,3,7,14$, and the lungs, livers, kidneys were removed for analysis. The dose of $10^{8} \mathrm{pfu}$ per mouse was used for later study since this dose caused minimal inflammation and provides high level of gene expression. At least 3 animals sacrificed at each of the aboveindicated days were used for each group. AGT mRNA was detected by real time PCR. Overexpression of AGT gene was confirmed by western blotting and immunohisto chemistry (IHC). Lung collagen content using picrosirius red staining and HP assay was measured. ISEL and histological features was examined. ${ }^{21}$

To test if those processes are affected by ANG II mediated apoptosis pathway, mice were also receive daily intraperitoneal injections of caspases inhibitor ZVAD at $1 \mathrm{mg} / \mathrm{kg}$ or intratracheal instillations of AT1 antagonist Losartan at dose of $250 \mathrm{ug} / \mathrm{mice}$ or nonselective angiotensin receptor antagonist Saralasin at dose of 50ug/ mice throughout the test interval, starting from one day before the adenoviral vector at the doses of $10^{8}$ pfu per mouse was administrated. At least 3 animals sacrificed at each of the above-indicated days were used for each group. 14 days after instillation of BLEO, at which point all animals were sacrificed for histology, detection of collagen or DNA fragmentation, and caspase 3 activation in epithelial cells.

\section{Surgical procedures}

Immediately before sacrifice, animals were given intraperitoneal injections of sodium pentobarbital and the trachea was cannulated. After perfusion by PBS, the left lung was ligated at the hilus, excised distal to the ligation, and immediately frozen in liquid N2 for hydroxyproline assay of total collagen (see below). The remaining lung tissues were carefully removed and were instilled with $4 \%$ paraformaldehyde in phosphate-buffered saline (PBS) at $20 \mathrm{~cm}$ of $\mathrm{H} 2 \mathrm{O}$ constant pressure, then immersed in the same fixative for 30 minutes followed by storage in $70 \%$ ethanol. The fixed tissues were washed with PBS three times for 15 minutes and were then embedded in paraffin. Five $\mu \mathrm{m}$ sections of lung were deparaffinized by passing through xylene, xylene: alcohol 1:1,100\% alcohol, and 70\% alcohol for 10 minutes each. Ethanol was removing by rinsing with distilled water.

\section{Identification and quantitation of apoptotic cells localization of DNA fragmentation by ISEL}

ISEL of fragmented DNA was conducted by a modification of the method of Mundle and colleagues. ${ }^{29}$ Briefly, ethanol was removed from deparaffinized lung sections by rinsing in distilled water for at least 10 minutes. The slides were then placed in $3 \%$ hydrogen peroxide (Sigma Chemical Co.) for 30 minutes at $20^{\circ} \mathrm{C}$, rinsed with PBS, and incubated with Proteinase K (Sigma) in standard saline citrate for 15 minutes at $37^{\circ} \mathrm{C}$. Samples were rinsed once in water, 
three times in $0.15 \mathrm{~mol} / \mathrm{L}$ PBS for 4 minutes each, and were then incubated in standard saline citrate $(0.3 \mathrm{~mol} / \mathrm{L} \mathrm{NaCl}$ and $30 \mathrm{mmol} / \mathrm{L}$ sodium citrate in water, $\mathrm{pH} \mathrm{7.0)}$ at $80^{\circ} \mathrm{C}$ for 20 minutes. After four rinses in PBS and four rinses in buffer A $(50 \mathrm{mmol} / \mathrm{L}$ Tris $/ \mathrm{HCl}$, $5 \mathrm{mmol} / \mathrm{L} \mathrm{MgCl}, 10 \mathrm{mmol} / \mathrm{L} \mathrm{B}$-mercaptoethanol, and $0.005 \%$ bovine serum albumin in water, $\mathrm{pH} 7.5$ ), the sections were incubated at $18^{\circ} \mathrm{C}$ for 2 hours with ISEL solution $(0.001 \mathrm{mmol} / \mathrm{L}$ digoxigenin-dUTP; 20U/ml DNA Polymerase I; and $0.01 \mathrm{mmol} / \mathrm{L}$ each of dATP, dCTP, and dGTP in buffer A). Afterward the sections were rinsed thoroughly five times with buffer A and three additional times in PBS. Detection of incorporated dUTP was achieved with by incubation for 2 hours at $37^{\circ} \mathrm{C}$ with AP-conjugated antidigoxigenin (Boehringer Mannheim) at 1/400 dilution. Bound AP-antibody was then detected with NBT chromogen system and the sections were mounted with Fluoromount solution (Southern Biotechnology, Birmingham, AL).

For quantitation of ISEL- positive epithelial cells, the number of positive cells within the surfaces of the alveolar walls was counted in a minimum of six randomly selected $\mathrm{x} 400$ microscopic fields per lung section. Positive cells within the alveolar airspaces, or otherwise clearly not within the surface of the alveolar wall, were not scored. The counts of positive nuclei per field were expressed as a percentage of the total number of nuclei in the same microscopic field. Sections from each of at least five rats per treatment group were analyzed by an investigator blinded to sample identity.

\section{Staining apoptotic cells on lung sections by TUNEL (terminal deoxynucleotidyl-UTP transferase- mediated nick end labeling)}

Cells containing fragmented DNA were identified by TUNEL through using apopTACS kit. Briefly, lung sections were deparaffinized by xyline and series ethanol, digested by proteinase $\mathrm{K}$, then incubated with the transferase and biotin labeled d-UTP mixture, followed by anti-biotin antibody incubation. A blue chromogen detection system was used, and lung sections were counterstained with eosin. For quantification, the numbers of positive nuclei were counted in a minimum of six randomly selected microscopic fields (40 X 10) per sample.

\section{Quantitation of lung collagen}

For quantitation of total lung collagen, tissues frozen in liquid $\mathrm{N} 2$ were dried to constant weight in preweighed tubes at $80^{\circ} \mathrm{C}$. The weighed dry tissue was hydrolyzed in $6 \mathrm{~N} \mathrm{HCl}$ and was subjected to determination of hydroxyproline as described earlier by Woessner. ${ }^{30}$ The efficiency of the hydrolysis was verified with rat-tail collagen by comparison to standard hydroxyproline (Sigma Chemical Co.).

\section{General histology examination}

The lung sections were stained by H-E methods and observed under light microscope for overall observation of wall thickness of airway, airway fibrosis, epithelial damage and vascularity and inflammatory cells infiltration like described before. ${ }^{21}$

\section{RNA isolation and reverse transcriptase polymerase chain reaction.}

Reverse transcriptase polymerase chain reaction (RT-PCR) was performed as described previously, ${ }^{23,31}$ and real-time RT-PCR was performed in the Physiology Department of Michigan State University. The annealing temperatures for PCR reactions were optimized for each prime by preliminary trials. The identity of the PCR products was determined by expected size in $1.6 \%$ agarose gels and by DNA sequencing of the PCR product excised from agarose gels (not shown). Total RNA was extracted from biopsies with TRI reagent (Molecular Research Center) according to the manufacturer's protocol. First-strand cDNA was synthesized from $1 \mu \mathrm{g}$ of total RNA with Superscript II reverse transcriptase (Invitrogen) and oligo (dT) $12-18$.

Real-time RT-PCR was performed with cDNA synthesized from 50ng of total RNA, SYBR Green PCR core reagents (Applied Biosystems, Foster City, CA) according to the manufacturer's instructions, and $0.2 \mu \mathrm{M}$ specific primers for human AGT (forward 5'GAG CAA TGA CCG CAT CAG-3' and reverse 5'-CAC AGC AAA CAG GAA TGG-3') and B-actin (forward 5'-AGG CCA ACC GCG AGA AGA TGA CC-3' and reverse 5' -gaa gtc cag ggc gac gta gc-3'), which produce PCR products of 151 and $332 \mathrm{bp}$, respectively(14). The PCR thermal profile started with 10-min activation of Taq polymerase at $95^{\circ} \mathrm{C}$ followed by 40 cycles of denaturation at $95^{\circ} \mathrm{C}$ for $30 \mathrm{~s}$, annealing at $55^{\circ} \mathrm{C}$ for $37 \mathrm{~s}$, and extension at $72^{\circ} \mathrm{C}$ for $37 \mathrm{~s}$, ending with dissociation curve analysis to validate the specificity of the PCR products. Reactions were performed in an Mx3000P machine (Stratagene, La Jolla, CA) and threshold cycle (CT) data were collected with MxPro-Mx3000P software version 3.0. The relative AGT expression was normalized to B-actin and calculated with the comparative CT method of $2^{-\Delta \Delta \mathrm{C}} \mathrm{T} .{ }^{14}$

\section{Immunohistochemistry (IHC) for active form of caspase 3 and AGT/ANGI}

IHC was performed with an antibody that recognizes only the active form of caspase 3 (BioVision, Mountain View, CA) and antiAGT/ANGI antibody (Santa Cruz, CA). Deparaffinized lung sections were blocked with a solution of 3\% bovine serum albumin in PBS for 1 hour; the primary antibody was then applied overnight at $4^{\circ} \mathrm{C}$ in $3 \%$ bovine serum albumin/PBS. After washing in PBS, the antibody was detected with a biotin-conjugated secondary antibody and avidinlinked chromogen system.

\section{Western blot analysis to detect and quantitate pulmonary AGT}

The lungs were perfused and snap-frozen in liquid nitrogen and stored at $-80^{\circ} \mathrm{C}$ until protein extraction. Lung protein extraction was performed as following. Briefly, the frozen lungs were pulverized in a chilled mortar and placed in NP40 tissue lysis buffer contain protease inhibitors cocktail. The samples were further centrifuged at $15,800 \mathrm{~g}$ for $10 \mathrm{~min}$ at $4^{\circ} \mathrm{C}$. The supernatants were collected, and protein levels were determined using a Bio-Rad protein assay (Bio-Rad Laboratories, Hercules, CA). The protein samples $(40 \mu \mathrm{g})$ were electrophoresed on $12 \%$ SDS-PAGE in a Mini-Protean II Electrophoresis Cell (Bio-Rad). Protein molecular weight markers (Invitrogen) were run parallel to each blot as an indicator of the molecular weight. The separated proteins were transferred at $150 \mathrm{~V}$ for 1.5 hours onto PVDF membrane (Bio-Rad) in a Mini Trans-Blot chamber with transfer buffer $(25 \mathrm{mM}$ Tris $\mathrm{HCl}, 192 \mathrm{mM}$ glycine, and $20 \%$ methanol). The PVDF membrane was blocked for $1 \mathrm{~h}$ using $5 \%$ no fat dry milk in Tris-buffered saline (TBS). For detection of AGT, a 1:100 dilution of ANG I/II antibody (Santa Cruz, CA) as used. After being washed, the membrane was incubated with horseradish peroxidase linked with the secondary antibody (anti-goat immunoglobulin G for AGT), as recommended by the manufacturer. Finally, the washed blots were exposed to an 
enhanced chemiluminescence (ECL) detection system (Amersham) and recorded on an autoradiograph (Amersham film).

\section{Results}

\section{Purified ANG II caused AEC apoptosis in vivo that was blocked by ATI antagonist losartan}

Our previous work showed that ANG II induces apoptosis in primary cultures of AECs by acting at AT1 receptor in vitro (24). If ANG II mediated AECs apoptosis is essential for pulmonary fibrogenesis, the first step we need to demonstrate is that intratracheal (I.T.) instillation of ANG can cause AECs apoptosis in vivo that could be blocked by AT1 receptor antagonist, losartan or the caspases inhibitor ZVADfmk. In our earlier report on Acute Lung Injury ${ }^{32}$ we showed that lung tissues from rats instilled with purified ANG II exhibited more fragmented DNA in nuclei (TUNEL Figure 1 in 40) and active caspase- 3 immunolabeling in bronchiolar epithelial cells and in AECs adjacent to alveolar ducts at 6 hours after instillation. Lung histology and detection of apoptosis in situ after intratracheal (I.T.) instillation of ANG II was also performed; control uninstalled lungs revealed occasional positive cells, but six hours after I.T. instillation of ANG II, significant numbers of TUNEL-positive and Caspase 3 -positive cells were observed, especially in regions adjacent to alveolar ducts. At higher magnification, many labeled cells were found in "corners" of the surface of alveolar walls, consistent with the location of alveolar epithelial type II cells (Figure 1). ${ }^{32}$ Quatitation of the TUNEL-positive and active Caspase 3-positive cells (Figure 2) ${ }^{32}$ revealed that instillation of the vehicle alone (PBS) did not induce detectible apoptosis, but $10 \mathrm{uM} \mathrm{ANG} \mathrm{II} \mathrm{increased} \mathrm{the} \mathrm{apoptotic}$ index of alveolar wall cells by 4.5 -fold when measured at 6 hours after the instillation. By 20 hours after instillation of ANG II, TUNEL had returned to the control value, presumably by a process involving epithelial repair. The increased TUNEL invoked by ANG II at 6 hours was attenuated by concurrent instillation of either the broad-spectrum Caspase inhibitor ZVADfmk (+ZVAD, $60 \mu \mathrm{M})$ or the angiotensin receptor subtype AT1-selective antagonist losartan $(+\mathrm{LOS}, 100 \mu \mathrm{M})$. Essentially identical results were obtained for immunolabeling of active Caspase-3; the same dose of losartan or ZVADfmk that blocked TUNEL also blocked the activation of Caspase 3.

\section{Purified ANG II induced collagen deposition in rat lungs both ex vivo and in vivo}

There is abundant evidence supporting that angiotensin system is involved in pulmonary fibrogenesis. No direct evidence was shown that upregulated ANG II level could directly cause pulmonary fibrosis. If AECs apoptosis is essential for pulmonary fibrogenesis, purified ANG II alone can cause fibrosis since ANG II induce AECs apoptosis both in vitro and in vivo (See above discussion of apoptosis data in $^{32}$ ). Intratracheal delivery of purified ANG II by aerosol daily would be a direct way to examine the role of the lung-derived ANG II in development of PF. Figure 1 Panel B shows that administration of aerosolized ANG II twice daily for 14 days caused a small but significant collagen accumulation in the lungs showed by HP assay, compared with control group (Figure 1 Panel $B, n=5, p<0.05$ versus control). Collagen amount in the lungs of animals exposed to aerosolized ANG II is increased by about $12 \%$ over animals only inhale the saline. In order to rule out the contribution of endocrine ANG system and blood pressure to the observed results, lung explants were processed to examine the profibrotic effect of ANG II. Figure
1 Panel A showed that there was more collagen accumulation in explants treated with ANG II which was blocked by SAR (saralasin), compared with control group treated without ANG II $(n=8, p<0.01$ versus control).

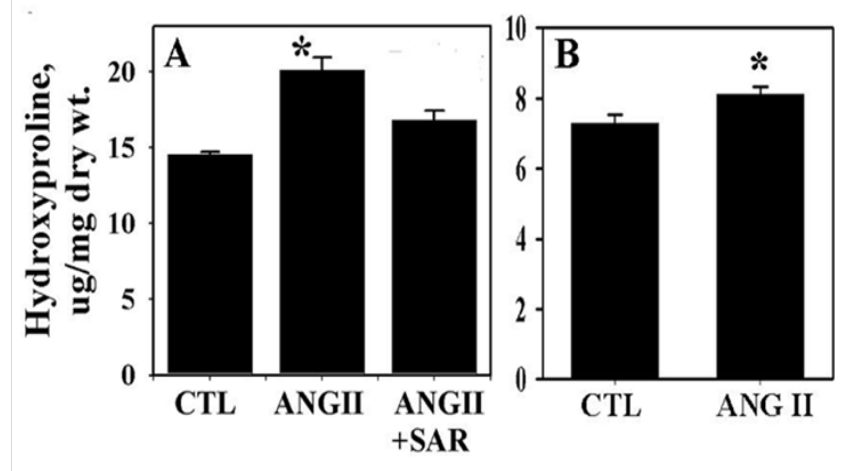

Figure I Purified ANG II induced collagen deposition in rat lungs both ex vivo and in vivo.

Panel A: Blood-depleted rat lung explants were treated with ANGII or vehicle in vitro. Collagen accumulation was measured by hydroxyproline (HP) assay (see Materials and Methods). SAR, saralasin. Bars are the mean \pm SEM of at least 4 separated samples; $*, p<0.0$ I versus control.

Panel B: Aerosolized ANG II was administered to adult rats by inhalation for 30 minutes daily for a total of 14 days (see Materials and Methods). Lung collagen accumulation was measured as described in A. Results were shown as $\mu g$ hydroxyproline (HP) per $\mathrm{mg}$ of dry lung tissue. (Bars are the mean S.E.M of at least 5 animals; $*=p<0.05$ versus control).
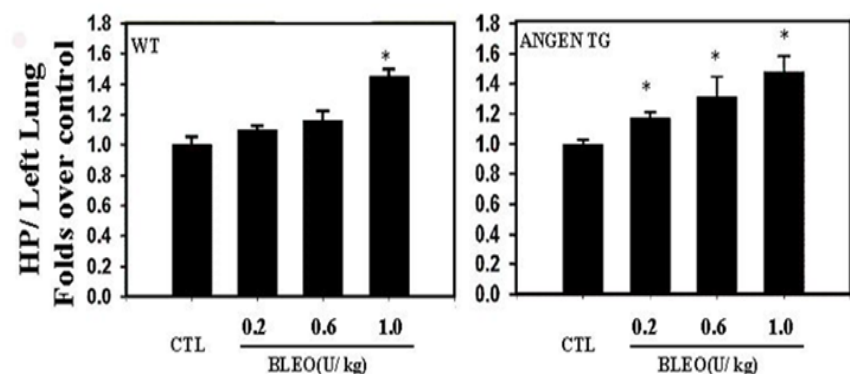

Figure 2 Ubiquitous overexpression of AGT enhanced bleomycin-induced pulmonary fibrosis in mice.

A single intratracheal bolus of bleomycin was administered to wild type (w.t) and AGT-overexpressing mice at suboptimal doses. Lung collagen accumulation was measured as described in Figure 4. Note significant increases in lung collagen at suboptimal bleomycin doses of 0.2 and $0.6 \mathrm{U} / \mathrm{kg}$ in AGT-overexpressing mice, but did not in WT mice. Bars are the mean S.E.M of at least 3 animals; $*, p<0.05$ vs. CTL.

\section{Ubiquitous overexpression of AGT enhanced BLEO- induced pulmonary fibrosis in mice}

In order to investigate the role of AGT in the development of pulmonary fibrosis, mice with ubiquitous overexpression of AGT were used in BLEO-induced pulmonary fibrosis, since those mice carry 4 copy of AGT gene and increase plasma AGT level by $25 \%$ over wild type (WT) mice. Figure 2 showed ubiquitous overexpression of AGT enhanced BLEO-induced pulmonary fibrosis in mice. Overexpression of AGT enhanced profibrogenic effect of bleomycin at suboptimal dose. BLEO induced significant increase of collagen amount in AGT 
overexpession mice at suboptimal doses 0.2 and $0.6 \mathrm{U} / \mathrm{kg}$, but did not in WT mice. BLEO had the same profibrotic effect by causing about $40 \%$ increase over corresponding control animals in both AGT overexpression and WT mice at dose $1 \mathrm{U} / \mathrm{kg}$. (Figure 3, P<0.05 VS CTL, $n=3)$. Those data suggest that AGT plays important role in the development of PF.

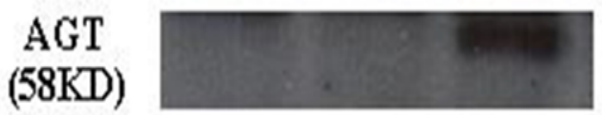

\section{CTL Ad-Null Ad-AGT}

Figure 3 Western blot for AGT protein after Ad-AGT delivery to mouse lung. Showed that AGT protein in the lungs, which were perfused by PBS to remove AGT in serum, 7 days after I.T. instillation of Ad-AGT virus at $10^{8}$ pfu per mouse was increased relative to control lungs instilled with saline or Ad-Null virus.

Overexpression of AGT in the mice lungs by intratracheal instillation of adenovirus carrying AGT gene

Due to the existence of the negative feedback system to compensate AGT upregulation, mice with systemic overexpression of AGT gene only has $25 \%$ higher plasma AGT level than WT mice although they carry 4 copies of AGT gene. ${ }^{27}$ However, local overexpression of AGT can avoid the systemic feedback loop and increase the AGT level in the lung. Adenoviral AGT gene delivery is chosen due to the long-term AGT expression (weeks), no requirement for repeated administration. Both mRNA and protein level of AGT was increased in $\mathrm{C} 57 \mathrm{BL} / 6 \mathrm{~J}$ mice lungs after intratracheal delivery of Ad-AGT in a dose and time dependent manner, starting from day 1 and lasting until day 14, peaking at day 7. In Figure 4, real time QPCR detected that the pulmonary AGT mRNA level, 14 days after I.T. instillation of Ad-AGT virus at $10^{8}$ pfu per mouse, were significantly higher than that in control lungs instilled with saline or Ad-Null virus $(\mathrm{P}<0.01 \mathrm{vs}$. CTL). In Figure 3, western blot for AGT protein showed that AGT protein in the lungs, which were perfused by PBS to remove AGT in serum, 7 days after I.T. instillation of Ad-AGT virus at $10^{8}$ pfu per mouse was increased relative to control lungs instilled with saline or Ad-Null virus. In Figure 5 Immunohistochemistry (IHC) staining by using anti-ANG I antibody that cross-reacted with AGT (see Figure 3 western blot) showed that at 14 days after intratracheal instillation of Ad-AGT at $10^{8}$ pfu per mouse, AGT/ANG I (Brown) was found in alveolar walls cell at the corners, consistent with the identity of those alveolar wall cells as type II AECs. No positive labeling was seen in saline (CTL) or Ad-Null virus treated mice lungs.

\section{Blockade of Ad-AGT virus caused pulmonary fibrosis by angiotensin II receptor antagonists and a caspases inhibitor}

In Figure 6, histology of mouse lungs was shown by hematoxylin and eosin preparations of mouse lung instilled intratracheally 14 days earlier with sterile saline (CTL), Ad-Null, or Ad-AGT (Original magnifications, x100). Exposure to Ad-AGT for 14 days resulted in typical histological changes of lung fibrosis. These include the infiltration of inflammatory cells, the obliteration of alveolar space, thickening of alveolar walls, and collagen accumulation (Ad-AGT), which were not observed in Ad-null or saline treated mice lungs (CTL and Ad-null). Figure 7 showed quantitation of total lung collagen by hydroxyproline (HP) assay. Administration of Ad-AGT at $10^{8}$ pfu per mouse caused significant collagen accumulation in the lungs at day 14 showed by HP assay, compared with control and Ad-null treated group. Results were shown as $\mu \mathrm{g}$ hydroxyproline (HP) per left lungs ( $\mathrm{N}=\mathrm{at}$ least 3; $\mathrm{p}<0.01)$. Caspases inhibitor ZVAD, AT1 antagonist Losartan, and non-selective angiotensin II receptor antagonist Saralasin blocked collagen accumulation in the lungs caused by overexpression of AGT.

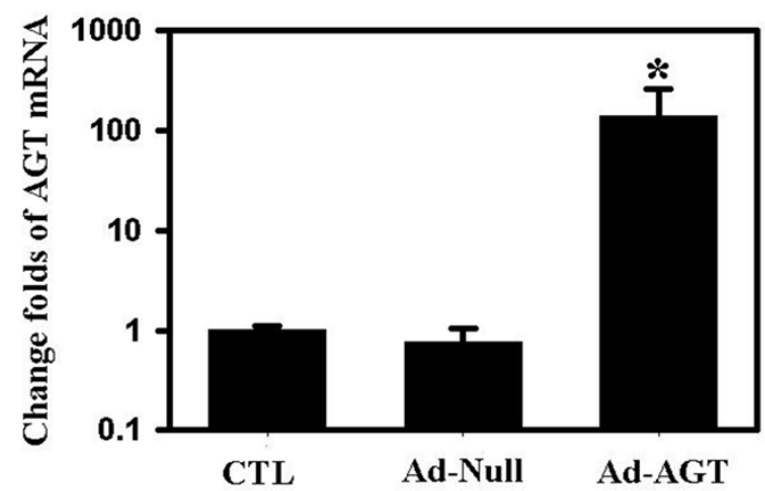

Figure 4 Overexpression of AGT mRNA in mouse lung by adenoviral delivery. Type $V$ adenoviruses expressing mouse AGT (Ad-AGT) or without transgene (Ad-null) were instilled I.T.at $10^{8}$ pfu per mouse. Total lung RNA was extracted 14 days later and subjected to realtime PCR for AGT mRNA (see Materials and Methods). Bars are the means $\pm S . E . M$ of $n=3, p<0.01$ vs. $C T L$ ).

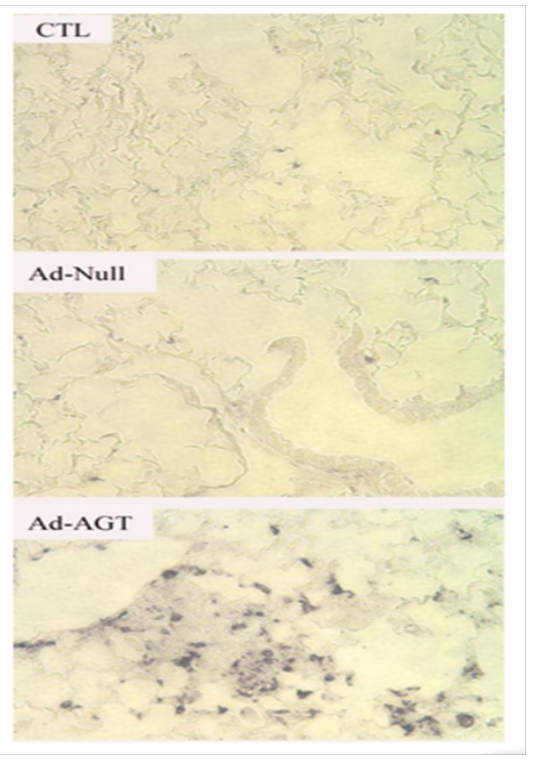

Figure 5 Immunohistochemistry (IHC) staining by using anti-ANG I antibody that cross-reacted with AGT (see Figure 4 western blot) showed that at 14 days after intratracheal instillation of Ad-AGT at $10^{8}$ pfu per mouse, AGT/ ANG I (Brown) was found in alveolar walls cell at the corners, consistent with the identity of those alveolar wall cells as type II AECs. No positive labeling was seen in saline $(C T L)$ or Ad-Null virus treated mice lungs. 


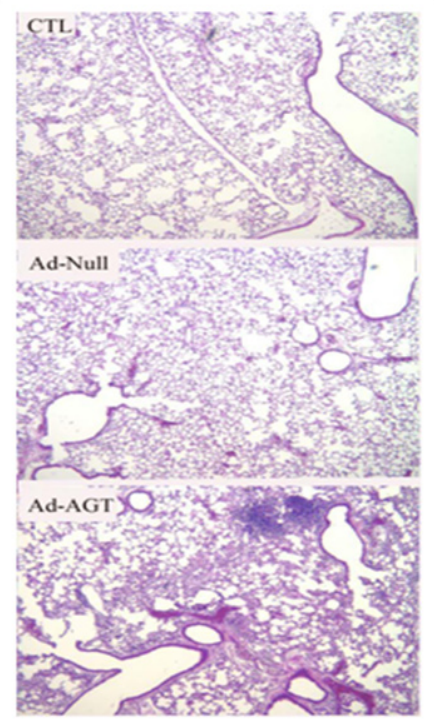

Figure 6 Histology of mouse lungs at 14 days after instillation of Ad-AGT. Hematoxylin and eosin preparations of mouse lung instilled intratracheally 14 days earlier with sterile saline (CTL), Ad-Null, or Ad-AGT (Original magnifications, $x 100$ ). Exposure to Ad-AGT for 14 days resulted in typical histological changes of lung fibrosis. These include the infiltration of inflammatory cells, the obliteration of alveolar space, thickening of alveolar walls, and collagen accumulation (Ad-AGT), which were not observed in Adnull or saline treated mice lungs (CTL and Ad-null).

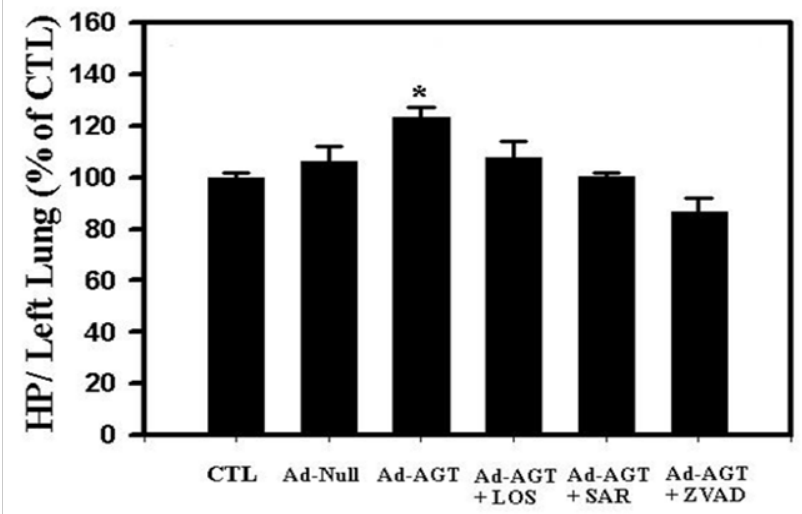

Figure 7 Quantitation of total lung collagen by hydroxyproline (HP) assay. Administration of Ad-AGT at $10^{8}$ pfu per mouse caused significant collagen accumulation in the lungs at day 14 showed by HP assay, compared with control and Ad-null treated group. Results were shown as $\mu \mathrm{g}$ hydroxyproline (HP) per left lungs (Bars are the mean SEM of at least 3 animals; *, $p<0.01$ ). Caspases inhibitor ZVAD, ATI antagonist Losartan, and non-selective ANGII receptor antagonist Saralasin blocked collagen accumulation in the lungs caused by overexpression of AGT.

\section{Caspases inhibitor ZVAD}

Figure 8 showed detection of apoptotic cells by in situ end labeling (ISEL) of fragmented DNA 14 days after described treatment. Male C57/BL mice received indicated treatment intratracheally; lung tissues were harvested 14 days after treatment and subjected to ISEL coupled to AP-NBT detection system (see METHODS). In CTL group, ISEL-positive nuclei (in blue color) were not observed. In the Ad-AGT group, ISEL-positive nuclei were observed in cells within the alveolar walls, many ISEL-positive nuclei were observed in septal wall cells at the alveolar corners. Administration of non-selective ANG II receptor antagonist Saralasin and Caspases inhibitor ZVAD decreased ISEL positive AECs cells in response to Ad-AGT, which has been demonstrated quantitatively in Figure $9(P<0.01)$.

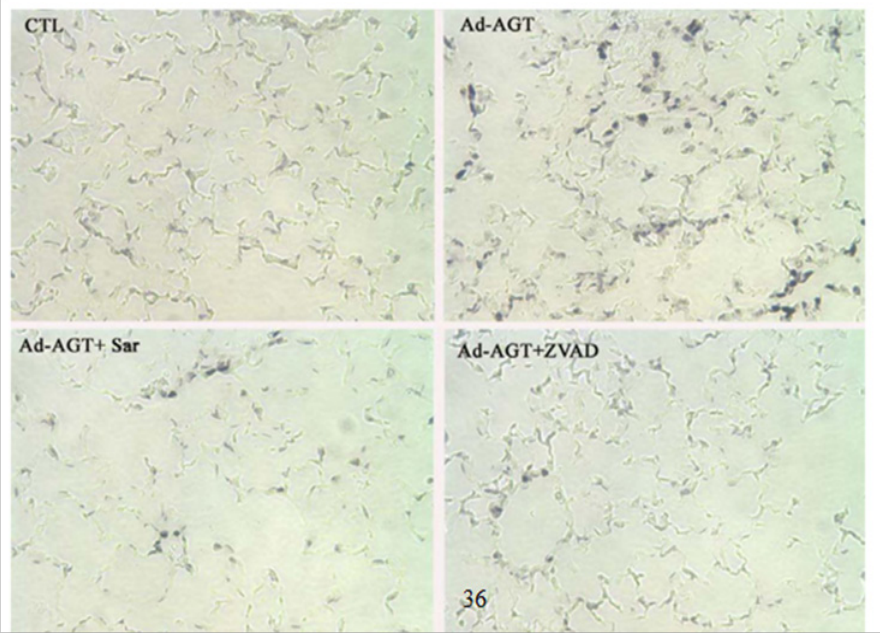

Figure 8 Detection of apoptotic cells by in situ end labeling (ISEL) of fragmented DNA 14 days after described treatment. Male C57/BL mice received indicated treatment intratracheally; lung tissues were harvested 14 days after treatment and subjected to ISEL coupled to AP-NBT detection system (see METHODS). Positive reaction is blue. In CTL group, ISEL-positive nuclei were not observed. In Ad-AGT group, ISEL-positive nuclei were observed in cells within the alveolar walls, many ISEL-positive nuclei were observed in septal wall cells at the alveolar corners. Administration of nonselective ANGIl receptor antagonist Saralasin and Caspases inhibitor ZVAD decreased ISEL positive cells in response to Ad-AGT.

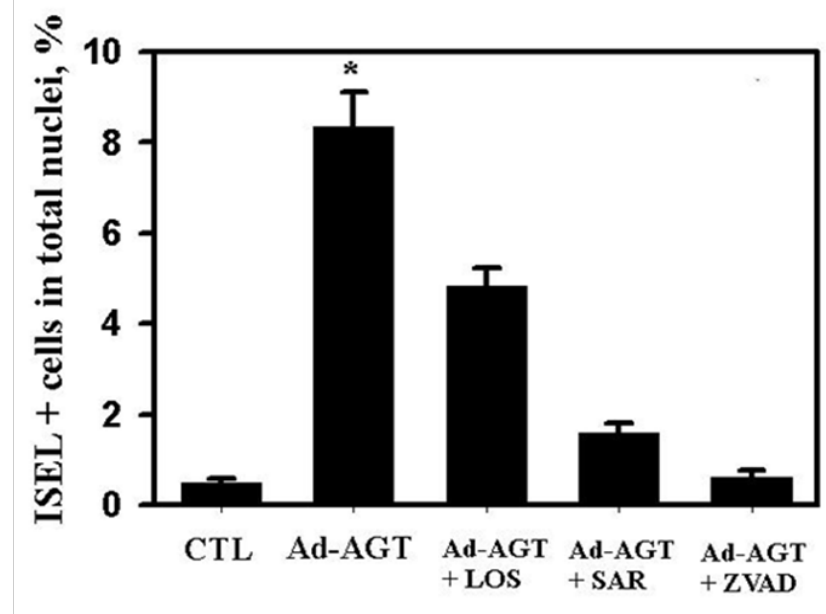

Figure 9 Inhibition of DNA fragmentation in lung epithelial cells 14 days after Ad-AGT instillation by ZVAD, Losartan and Saralasin. Positive labeling was scored in cells within the alveolar septal wall surfaces (Bars are the means \pm SEM of at least 3 animal; $*, P<0.01$ ).

\section{Discussion}

\section{Local angiotensin system in pulmonary fibrosis}

Local angiotensin system is defined as ANG II is largely, if not completely, synthesized locally in certain tissue or organs, in 
additional of diffusion and/or receptor-mediated uptake of circulating ANGII. ${ }^{14}$ Recently a few studies suggested the existence of local angiotensin systems in the lung especially with pulmonary fibrosis. ${ }^{33}$

Previous work suggests that pulmonary angiotensin system plays an important role in the development of pulmonary fibrosis. Marshall and our data showed that pulmonary ANG II is elevated in bleomycin-induced pulmonary fibrosis model. ${ }^{12,13}$ Our recently results demonstrated that AGT, the only precursor of ANG II, is increased in the type II AECs (AECs) and myofibroblasts at both mRNA level and protein level, and its expression is related with apoptosis of AECs and required in the development of bleomycin-induced pulmonary fibrosis, ${ }^{12}$ and similar finding was observed in IPF patients. ${ }^{14}$ In addition, the converting enzymes for synthesis of angiotensin peptides like Cathepsin D, angiotensin converting enzyme (ACE), and AT1 receptors were upregulated in both animal and human fibrotic lung. Furthermore, Angiotensin system antagonists like ACE inhibitors and AT1 selective antagonists blocked experimental lung fibrosis. However, the causative effect of pulmonary angitensin system on pulmonary fibrosis is not clear. Therefore, we hypothesize that overexpression of AGT or elevation of pulmonary ANG II level in the lung will cause apoptosis of AECs and pulmonary fibrosis.

\section{Direct effect of purified ANG II on apoptosis of AECs and pulmonary fibrosis}

There is abundant evidence as listed above supporting that angiotensin system is involved in pulmonary fibrogenesis. No direct evidence was shown that upregulated ANG II level could directly cause pulmonary fibrosis. Since apoptosis of AECs is considered as the initial step for pulmonary fibrogenesis, ${ }^{34}$ we first test if intratracheal (I.T.) instillation of ANG II can cause AECs apoptosis in vivo that could be blocked by AT1 receptor antagonist, losartan or the caspases inhibitor ZVAD fmk, as it did in vitro on AECs. ${ }^{31}$ Previous studies of primary cultures of type II AECs showed that ANG II induces apoptosis in vitro in a dose-dependent manner with maximal stimulation at $10 \mathrm{uM} \cdot{ }^{31,35}$ For this reason, the single concentration of $10 \mathrm{uM}$ ANG II was tested for the ability to induce AECs apoptosis in vivo after I.T. instillation performed by standard methods.

In our earlier study $(\mathrm{x})$, we used two independent assays (TUNEL and active caspase- 3 immunolabeling) to confirm that ANG II can cause AECs apoptosis in vivo. Detection of fragmented DNA by TUNEL revealed significant numbers of TUNEL-positive cells, particularly near alveolar ducts, six hours after I.T. ANG II. Increased TUNEL-positive signal and active caspase-3 expression in vivo were abrogated by concurrent instillation of a caspase inhibitor (ZVAD $60 \mathrm{uM}$ ) or by an ANG II type I receptor antagonist (Losartan 100uM). Apoptosis detectible at 6 hours post-instillation was resolved by 20 hours, a time sufficient for epithelial repair by cell proliferation; this finding may reflect the short biological half-life of the ANG II. Regardless, the induction of apoptosis by 6hour is consistent with the work of Hagimoto et al., 5 who found that pulmonary epithelial apoptosis induced by I.T. instillation of bleomycin also was detectible by 6 hours post-instillation. , $^{5}$

If AECs apoptosis is essential for pulmonary fibrogenesis, purified ANG II alone can cause fibrosis since ANG II induce AECs apoptosis both in vitro and in vivo, but the finding that apoptosis detectible at 6 hours post-instillation of ANG II was resolved by 20 hours made us use alternative strategy to administrate purified ANG II repeatedly by aerosol twice daily for 14days which is the minimum time needed to develop pulmonary fibrosis. Administration of aerosolized
ANG II caused a small but significant collagen accumulation in the lungs showed by HP assay, compared with control group $(\mathrm{p}<0.05$ versus control). Collagen amount in the lungs of animals exposed to aerosolized ANG II is increased by about $12 \%$ over animals only inhale the saline, but in lung explant, ANG II caused about $30 \%$ increase of collagen accumulation (Figure 1). The difference in profibrotic effect of ANG II between in vivo model and ex vivo explant may be due to ANG II has longer biological half-life in culture medium than in whole animal model. Nevertheless, Saralasin blocked ANG II induced collagen accumulation in explant, indicating that ANG II is responsible for the profibrotic effect.

\section{Rate-limiting step of ANG II generation in pulmonary fibrosis}

AGT is the only known precursor for the synthesis of angiotensin peptides. Circulating levels of AGT are approximately equal to the $\mathrm{Km}$ of renin for its substrate (about $1 \mathrm{uM}){ }^{36}$ Therefore, the rate of ANG II synthesis can be regulated by changes in AGT levels. Since the normal concentration of AGT is near the $\mathrm{Km}$ for its reaction with renin, ${ }^{36}$ one would expect any change in AGT levels to be accompanied by parallel changes in the formation and actions of Ang II. For instance, transgenic mice expressing the rat AGT gene are hypertensive ${ }^{37}$ and mice without AGT gene expression are hypotensive. ${ }^{38}$ In addition, for any given level of renin activity ANG II synthesis can be altered by changes in the concentration of available AGT. For example, up-regulation of AGT in tissue can alter tissue concentrations of ANG II, particularly, in tissues not subjected to systemic short loop or long loop feedback control..$^{39}$ In bleomycin induced pulmonary fibrosis model, AGT expression is upregulated in the lung more than 2 fold. ${ }^{16}$ Elevation of local pulmonary AGT concentration increased pulmonary ANG II concentration. ${ }^{12}$ Furthermore, intratracheal delivery of antisense against AGT mRNA blocked the apoptosis of AECs and subsequent fibrosis, which is consistent with the result that local ANG II generation is decreased. ${ }^{12}$ Similar finding was observed in human IPF patients. ${ }^{14}$ AGT mRNA was found to be 21 -fold more abundant in IPF lung tissue relative to the control specimens of human lung and AGT protein in IPF lung biopsies is about 3.6-fold higher levels of compared to nonfibrotic lung. ${ }^{14}$

Those data may explain why those mice with ubiquitous overexpression of AGT do not have pulmonary fibrosis since mice with systemic overexpression of AGT gene only has $25 \%$ higher plasma AGT level than WT mice although they carry 4 copies of AGT gene, due to the existence of the negative feedback system to compensate AGT upregulation. ${ }^{27}$ Still the slightly elevated AGT in mice with ubiquitous overexpression of AGT contributed to the development of pulmonary fibrosis since overexpression of AGT enhanced profibrogenic effect of bleomycin at suboptimal dose (Figure 2). In contrast, local overexpression of AGT by adenoviral AGT gene delivery can avoid the systemic feedback loop and increase the AGT mRNA level in the lung about 100 folds by Ad-AGT virus at the dose of $10^{8} \mathrm{pfu}$ per mouse (Figure 3). This dose is used for later study since this dose caused minimal inflammation and provides high level of gene expression. Adenovirus encoded- AGT protein only expressed in lungs treated with the adenoviral AGT gene delivery (Figure 4), although in saline or null adenovirus treated lung, there are some basic line endogenous AGT expression (data not shown). The western results were confirmed by immunolabeling of AGT/ ANG I protein in Figure 5. 14 days after intratracheal instillation of Ad-AGT at $10^{8}$ pfu per mouse, AGT/ANG I was found primarily in alveolar walls cell at the corners, consistent with the identity of those 
alveolar wall cells as type II AECs, and other cell types with unknown identification. From our previous studies in bleomcin induced experimental pulmonary fibrosis animal model and human IPF, ${ }^{12,14}$ at least two cell types found in the fibrotic lungs, myofibroblasts and AEC undergoing apoptosis, were capable of synthesizing ANG peptides de novo. So the immunolabeling of AGT/ANG I we observed at 14 days after intratracheal instillation of Ad-AGT might be the combination of Adenoviral encoded-AGT and de novo synthesis of AGT from myofibroblasts and AEC undergoing apoptosis since at 14 day after intratracheal instillation of Ad-AGT at $10^{8}$ pfu per mouse caused pulmonary fibrosis confirmed by histology and HP assay (Figure 6) (Figure 7). The picrosirius red technique was also used to identify lung collagen. More extensive collagen accumulation in alveolar septa and peribronchial parenchyma were observed in animals treated with AdAGT for 14 days, compared to saline or Ad-Null treated mice (data not shown). Those data indicate that selectively overexpression of AGT, the precursor of ANG II, in the lungs by intratracheal instillation of adenovirus carrying AGT gene is sufficient for the fibrogenesis and other local angiotensin system components like converting enzymes such as Cathepsin D, Angiotensin I converting Enzymes (ACE) do not need to be activated even though those enzymes are upregulated in human and animal experimental lung fibrosis, suggesting that pulmonary AGT level is the rate-limiting step of ANG II generation in pulmonary fibrosis.

To test if selective overexpression of AGT in the lung by I.T. instillation of Ad-AGT induced pulmonary firbosis is affected by ANG II mediated apoptosis pathway, mice were also receive daily intraperitoneal injections of caspases inhibitor ZVAD or intratracheal instillations of AT1 antagonist Losartan or non-selective angiotensin receptor antagonist Saralasin. Figure 7-9 showed that the level of AGT expression correlates with the apoptosis of AECs and fibrosis in the lung. Successful blockade of Ad- AGT induced pulmonary fibrosis by the caspase inhibitor ZVADfmk supports the contention that apoptosis of AECs was a causal factor in the fibrogenesis. Furthermore, blockade of Ad- AGT induced apoptosis of AECs and pulmonary fibrosis by the AT1 antagonist Losartan or non-selective angiotensin receptor antagonist Saralasin suggests that Ad- AGT induced apoptosis of AECs and pulmonary fibrosis is ANG II dependent.

It seems Saralasin had better efficacy in term of blockade of apoptosis of AECs and pulmonary fibrosis than Losartan in present study, suggesting that both AT1 and AT2 receptors are responsible for overexpression of AGT induced apoptosis of AECs and pulmonary fibrosis, but AT1 play a major role since Losartan also blocked apoptosis of AECs and pulmonary fibrosis, which are consistent with our previous studies. The function of the AT2 receptor in the development of pulmonary fibrosis caused by overexpression of AGT need to be further investigated.

\section{Summary and conclusions}

This is the first study to demonstrate the causative effect of elevated pulmonary angiotensin II and AGT overexpression on development of pulmonary fibrosis. We demonstrated that purified ANG II was administrated to elevate the local ANG II level in the lung, and caused significant collagen accumulation in the lungs both ex vivo and in vivo. I.T. delivery of Ad-AGT overexpressed AGT primarily in the lung and caused apoptosis of AECs and pulmonary fibrosis that can be blocked by Caspases inhibitor ZVAD, AT1 antagonist Losartan, and non-selective angiotensin II receptor antagonist Saralasin, indicating causative effect of elevated pulmonary AGT on pulmonary fibrosis is mediated by ANG II -induced AECs apoptosis. In conclusion, elevated pulmonary AGT and/or ANG II play a causative role in the development of pulmonary fibrosis.

\section{Grants}

This work was supported by National Heart, Lung, and Blood Institute Grant HL-45136 and by the American Physiological Society.

\section{Acknowledgments}

None.

\section{Conflicts of interest}

The authors declare that there is no conflict of interest regarding the publication of this paper.

\section{References}

1. Fonseca C, Abraham D, Black CM. Lung fibrosis. Springer Semin Immunopathol. 2000;21(4):453-474.

2. Crystal RG, Bitterman PB, Mossman B, et al. Future research directions in idiopathic pulmonary fibrosis: summary of a National Heart, Lung, and Blood Institute working group. Am J Respir Crit Care Med. 2002;166(2):236-246.

3. Mason RJ, Schwarz MI, Hunninghake GW, et al. NHLBI Workshop Summary. Pharmacological therapy for idiopathic pulmonary fibrosis. Past, present, and future. Am J Respir Crit Care Med. 1999;160(5 Pt 1):1771-1777.

4. Uhal BD, Joshi I, Hughes WF, et al. Alveolar epithelial cell death adjacent to underlying myofibroblasts in advanced fibrotic human lung. Am J Physiol. 1998;275(6 Pt 1):L1192-L1199.

5. Hagimoto N, Kuwano K, Nomoto Y, et al. Apoptosis and expression of Fas/Fas ligand mRNA in bleomycin-induced pulmonary fibrosis in mice. Am J Respir Cell Mol Biol. 1997;16(1):91-101.

6. Hagimoto N, Kuwano K, Miyazaki H, et al. Induction of apoptosis and pulmonary fibrosis in mice in response to ligation of Fas antigen. Am J Respir Cell Mol Biol. 1997;17(3):272-278.

7. Kuwano K, Kunitake R, Maeyama T, et al. Attenuation of bleomycininduced pneumopathy in mice by a caspase inhibitor. Am J Physiol Lung Cell Mol Physiol. 2001;280(2):L316-L325.

8. Wang R, Ibarra-Sunga O, Verlinski L, et al. Abrogation of bleomycininduced epithelial apoptosis and lung fibrosis by captopril or by a caspase inhibitor. Am J Physiol Lung Cell Mol Physiol. 2000;279(1):L143-L151.

9. Selman M, King TE, Pardo A. Idiopathic pulmonary fibrosis: prevailing and evolving hypotheses about its pathogenesis and implications for therapy. Ann Intern Med. 2001;134(2):136-151.

10. Kuwano K, Kawasaki M, Maeyama T, et al. Soluble form of fas and fas ligand in BAL fluid from patients with pulmonary fibrosis and bronchiolitis obliterans organizing pneumonia. Chest. 2000;118(2):451-458.

11. Kuwano K, Kunitake R, Kawasaki M, et al. P21Waf1/Cip1/Sdi1 and p53 expression in association with DNA strand breaks in idiopathic pulmonary fibrosis. Am J Respir Crit Care Med. 154(2 Pt 1):477-483.

12. Li X, Zhuang J, Rayford $\mathrm{H}$, et al. Attenuation of bleomycin-induced pulmonary fibrosis by intratracheal administration of antisense oligonucleotides against angiotensinogen mRNA. Curr Pharm Des. 2007;13(12):1257-1268.

13. Marshall RP, Gohlke P, Chambers RC, et al. Angiotensin II and the fibroproliferative response to acute lung injury. Am J Physiol Lung Cell Mol Physiol. 2004;286(1):L156-L164. 
14. Li X, Molina-Molina M, Abdul-Hafez A, et al. Extravascular sources of lung angiotensin peptide synthesis in idiopathic pulmonary fibrosis. $\mathrm{Am}$ J Physiol Lung Cell Mol Physiol. 2006;291(5):L887-L895.

15. Kasper M, Lackie P, Haase M, et al. Immunolocalization of cathepsin $\mathrm{D}$ in pneumocytes of normal human lung and in pulmonary fibrosis. Virchows Arch. 1996;428(4-5):207-215.

16. Koslowski R, Knoch K, Kuhlisch E, et al. Cathepsins in bleomycininduced lung injury in rat. Eur Respir J. 2003;22(3):427-435.

17. Specks U, Martin WJ, 2nd, and Rohrbach MS. Bronchoalveolar lavage fluid angiotensin-converting enzyme in interstitial lung diseases. $\mathrm{Am}$ Rev Respir Dis. 1990;141(1):117-123.

18. Venkatesan N, Punithavathi V, Chandrakasan G. Curcumin protects bleomycin-induced lung injury in rats. Life Sci. 1997;61(6):PL51-PL58.

19. Otsuka M, Takahashi H, Shiratori M, et al. Reduction of bleomycin induced lung fibrosis by candesartan cilexetil, an angiotensin II type 1 receptor antagonist. Thorax. 2004;59(1):31-38.

20. Renzoni EA, Abraham DJ, Howat S, et al. Gene expression profiling reveals novel TGFbeta targets in adult lung fibroblasts. Respir Res. 2004;5:24.

21. Li X, Rayford H, Uhal BD. Essential roles for angiotensin receptor AT1a in bleomycin-induced apoptosis and lung fibrosis in mice. Am J Pathol. 2003;163(6):2523-2530.

22. Molteni A, Moulder JE, Cohen EF, et al. Control of radiation-induced pneumopathy and lung fibrosis by angiotensin-converting enzyme inhibitors and an angiotensin II type 1 receptor blocker. Int $J$ Radiat Biol. 2000;76(4):523-532.

23. Molteni A, Ward WF, Ts'ao $\mathrm{CH}$, et al. Monocrotaline-induced cardiopulmonary damage in rats: amelioration by the angiotensinconverting enzyme inhibitor CL242817. Proc Soc Exp Biol Med. 1986;182(4):483-493.

24. Uhal BD, Wang R, Laukka J, et al. Inhibition of amiodarone-induced lung fibrosis but not alveolitis by angiotensin system antagonists. Pharmacol Toxicol. 2003;92(2):81-87.

25. Ward WF, Molteni A, Ts'ao $\mathrm{CH}$, et al. Captopril reduces collagen and mast cell accumulation in irradiated rat lung. Int $J$ Radiat Oncol Biol Phys. 1990;19(6):1405-1409.

26. Taylor BK, Stoops TD, Everett AD. Protein phosphatase inhibitors arrest cell cycle and reduce branching morphogenesis in fetal rat lung cultures. Am J Physiol Lung Cell Mol Physiol. 2000;278(5):L1062-L1070.
27. Kim HS, Krege JH, Kluckman KD, et al. Genetic control of blood pressure and the angiotensinogen locus. Proc Natl Acad Sci USA. 1995;92(7):2735-2739.

28. Kolb M, Bonniaud P, Galt T, et al. Differences in the fibrogenic response after transfer of active transforming growth factor-betal gene to lungs of "fibrosis-prone" and "fibrosis-resistant" mouse strains. Am J Respir Cell Mol Biol. 2002;27(2):141-150.

29. Mundle S, Iftikhar A, Shetty V, et al. In situ end labeling of DNA to detect apoptotic cell death in a variety of human tumors. Cell Death Differ. 1994;1(2):117-122.

30. Woessner J. The determination of hydroxyproline in tissue and protein samples containing small proportions of this imino acid. Arch Biochem Biophys. 1961;93:440-447.

31. Papp M, Li X, Zhuang J, et al. Angiotensin receptor subtype AT(1) mediates alveolar epithelial cell apoptosis in response to ANG II. Am J Physiol Lung Cell Mol Physiol. 2002;282:L713-L718.

32. Zhuang JJ, Li XP, Uhal BD, et al. Apoptosis-dependent acute pulmonary injury after intratracheal instillation of angiotensin II. Sheng Li Xue Bao. 2008;60(6):715-722.

33. Marshall RP. The pulmonary renin-angiotensin system. Curr Pharm Des. 2003;9(9):715-722.

34. Uhal BD. Epithelial apoptosis in the initiation of lung fibrosis. Eur Respir J Suppl. 2003;44:7s-9s.

35. Wang R, Zagariya A, Ibarra-Sunga O, et al. Angiotensin II induces apoptosis in human and rat alveolar epithelial cells. Am J Physiol. 1999;276(5 Pt 1):L885-L889.

36. Gould AB, Green D. Kinetics of the human renin and human substrate reaction. Cardiovasc Res. 1971;5(1):86-89.

37. Kimura S, Mullins JJ, Bunnemann B, et al. High blood pressure in transgenic mice carrying the rat angiotensinogen gene. Embo $\mathrm{J}$. 1992;11(3):821-827.

38. Tanimoto K, Sugiyama F, Goto Y, et al. Angiotensinogen-deficient mice with hypotension. J Biol Chem. 1994;269(50):31334-31337.

39. Poulsen $\mathrm{K}$ and Jacobson J. Enzymatic reactions of the renin-angiotensin system. In: Robertson JIS, Nicholls MG, editors. The renin- angiotensin system: Biochemistry and Physiology; 1993;5:1-12. 